\title{
Energy model for the Zr-based metallic glass alloy melt with clusters
}

\author{
YANG YuanSheng ${ }^{\dagger}$, LI HuiQiang \& TONG WenHui \\ Institute of Metal Research, Chinese Academy of Sciences, Shenyang 110016, China
}

An energy model for the melt of bulk metallic glass (BMG) with clusters was established, the Gibbs free energy and interfacial energy for the Zr-Al-Ni ternary alloy melt with $\mathrm{Zr}_{2} \mathrm{Ni}$ clusters were calculated, and the effects of the clusters on the Gibbs free energy, interfacial energy and nucleation rate were analyzed. The results showed that the existence of the clusters in the Zr-Al-Ni ternary alloy melt enables the Gibbs free energy to decrease in the composition range where bulk metallic glass forms easily, makes the interfacial energy increase and changes the distribution of the interfacial energy with the alloy composition. Because of the clusters in the melt, the Gibbs free energy of the $\mathrm{Zr}_{66} \mathrm{Al}_{8} \mathrm{Ni}_{26}$ alloy melt decreases about $0.3-1$ $\mathrm{kJ} / \mathrm{mol}$ and the interfacial energy between the melt and crystal nucleus increases about $0.016 \mathrm{~J} / \mathrm{m}^{2}$. The nucleation rate of the undercooled $\mathrm{Zr}_{66} \mathrm{Al}_{8} \mathrm{Ni}_{26}$ alloy melt decreases evidently under the influence of the clusters on Gibbs free energy and the interfacial energy, and the maximum of the nucleation rate in the melt with the $\mathrm{Zr}_{2} \mathrm{Ni}$ clusters is only about $10^{7} \mathrm{~mol}^{-1} \cdot \mathrm{s}^{-1}$.

bulk metallic glass, cluster, Gibbs free energy, interfacial energy

\section{Introduction}

Bulk metallic glass (BMG) has been widely studied because of its outstanding mechanical, physical and chemical properties. A series of Pd-, Zr-, La-, Mg- and Cu-based BMG are successfully prepared, but the mechanism of BMG formation has not been fully understood, which is still one of more interesting topics. Turnbull ${ }^{[1]}$ first proposed the classical theory for amorphous transformation in which it was considered that the detectable crystallization can be avoided if melt is cooled at a sufficiently high rate. On the basis of experiments, Uhlmann ${ }^{[2]}$ proposed the well-known criterion for glass formation that the volume fraction of the crystalline phase in metallic glass alloy is less than $10^{-6}$. According to the classical nucleation theory, the nucleation is dependent on the energy change of the melt which is considered one of the most important parameters during nucleation process. So, a lot of theoretical models and empirical models have been

Received February 13, 2007; accepted May 29, 2007

doi: $10.1007 / \mathrm{s} 11433-007-0048-4$

${ }^{\dagger}$ Corresponding author (email: ysyang@imr.ac.cn)

Supported by the National Natural Science Foundation of China (Grant No. 50395100) 
established for calculating the free energy of melt ${ }^{[3-5]}$. Because of the high cooling rate of $10^{2}-10^{3}$ $\mathrm{K} / \mathrm{s}$, deeply undercooling phenomenon will happen and a lot of clusters can be formed spontaneously in the melt during BMG preparation process ${ }^{[6]}$. Frank ${ }^{[7]}$ pointed out that the icosahedron has the lowest energy per atom of all clusters. In recent years, the clusters are quantitatively described and some microscopic approaches such as initio calculation ${ }^{[8]}$, Monte Carlo ${ }^{[9]}$ and molecular dynamics ${ }^{[10]}$ have been used to investigate the effect of clusters on the change of melt energy during BMG formation. Desré ${ }^{[11]}$ studied the probability of component undulation (cluster) in the melt by Gaussian distribution, considering the melt as the system composed of particles. Chen et al. ${ }^{[12]}$ proposed the thermodynamic model of multicomponent chemical short-range order (MCSRO) domain and their calculation results show that the appearance of clusters decreases the free energy of melt and increases the stability of the melt, thus promoting the formation of metallic glass. Holland-Moritz ${ }^{[13]}$ considered that the clusters with the short-range order (SRO) in the undercooled melt can influence the solid-liquid interfacial energy. However, very little has been reported on the effect of the cluster on the Gibbs energy of the metallic glass alloys, especially on that of the Zr-based alloys up to date. This paper establishes an energy model for the melt of bulk metallic glass with clusters, calculates the Gibbs free energy and interfacial energy for the Zr-Al-Ni ternary alloy melt with $\mathrm{Zr}_{2} \mathrm{Ni}$ clusters, and analyzes the effects of the clusters on the Gibbs free energy, interfacial energy and nucleation rate.

\section{The energy model for calculation}

\subsection{Gibbs energy of melt with clusters}

The Gibbs energy of liquid alloy is expressed as the sum of two contributions for convenience of calculation, one from the clusters and the other from the bulk melt. The interaction between the bulk melt and the clusters is weak and can be ignored.

If there are $n$ kinds of clusters in a multicomponent alloy system, and the number of clusters with the same microstate is unlimited, the liquid alloy is regarded as the Boltzmann system which is described as

$$
a_{1}=\omega_{1} \mathrm{e}^{-\alpha} \mathrm{e}^{-\frac{n}{R T} \Delta G_{f}\left(C, C_{0}\right)},
$$

and the mole fraction $f_{i}$ of the $i$ th cluster in one mole melt with the $C$ component is

$$
f_{i}=\int \frac{n}{Z} \mathrm{e}^{-n \frac{\Delta G_{f}\left(C, C_{0}\right)}{R T}} \mathrm{~d} n,
$$

where $a_{1}$ is the probability of cluster formation, $\omega_{1}$ is the microscopic state of the cluster, $C_{0}$ is the average composition of the melt, $C$ is the composition of the cluster, $n$ is the number of atoms in the cluster, $R$ is the gas constant, $Z$ is the collision frequency, $\Delta G_{f}\left(C, C_{0}\right)$ is the isothermal Gibbs energy for formation of one mole cluster in the melt.

The Gibbs energy of the system, $G$, is written as

$$
G=\sum_{i=1}^{n} f_{i} G_{i}+\left(1-\sum_{i=1}^{n} f_{i}\right) G_{1}+R T\left[\sum_{i=1}^{n} f_{i} \ln f_{i}+\left(1-\sum_{i=1}^{n} f_{i}\right) \ln \left(1-\sum_{i=1}^{n} f_{i}\right)\right],
$$

where $G_{i}$ is the molar Gibbs energy of the clusters and $G_{1}$ is the molar Gibbs energy of the residual melt ${ }^{[5]}$. 


\subsection{Interfacial energy}

The interfacial energy between the melt and crystal nucleus in the alloy melt, $\gamma_{\mathrm{SL}}$, can be calculated using the non-structural model as follows ${ }^{[14]}$ :

$$
\begin{gathered}
\gamma_{\mathrm{SL}}^{H}=\left[\left(\frac{V_{\mathrm{L}}}{V_{\mathrm{S}}}\right)^{2 / 3}-1\right] \sum_{i=1}^{n} x_{i}^{\prime}\left(\gamma_{i}^{\mathrm{L}}+\frac{x_{i}^{\prime}}{\sqrt[3]{V_{\mathrm{S}}^{2} N_{\mathrm{A}}}} R T \ln \frac{a_{i}^{\prime}}{a_{i}^{\mathrm{L}}}\right)+\left(\int_{T_{\mathrm{m}}}^{T}\left(C_{p, \mathrm{~L}}-C_{p, \mathrm{~S}}\right) \mathrm{d} T\right)\left(\frac{1}{4 \sqrt[3]{V_{\mathrm{S}}^{2} N_{\mathrm{A}}}}\right), \\
\gamma_{\mathrm{SL}}^{S}=\frac{\left(\Delta S_{\mathrm{f}}+\int_{T_{\mathrm{m}}}^{T} \frac{C_{p, \mathrm{~L}}-C_{p, \mathrm{~S}}}{T} \mathrm{~d} T-3 r R \ln \frac{V_{\mathrm{L}}+V_{\mathrm{S}}}{2 V_{\mathrm{S}}}\right) T}{\sqrt[3]{V_{\mathrm{S}}^{2} N_{\mathrm{A}}}},
\end{gathered}
$$

where $\gamma_{\mathrm{SL}}^{H}, \gamma_{\mathrm{SL}}^{S}$ are the interfacial energies from the enthalpy and the entropy, respectively; $V_{\mathrm{L}}, V_{\mathrm{S}}$ are the molar volumes of the liquid and solid, respectively; $C_{p, \mathrm{~L}}, C_{p, \mathrm{~S}}$ are the specific heat of the liquid and solid, respectively; $x_{i}^{\mathrm{L}}, x_{i}^{\prime}$ are the molar fraction of the $i$ th element in the liquid and in the interface, respectively; $\Delta S_{\mathrm{f}}$ is the entropy of fusion, $N_{\mathrm{A}}$ is the Avogadro constant, $\gamma_{i}^{\mathrm{L}}$ is the liquid-vapor interfacial energy of the $i$ th element (pure metal); $r$ is Grüneisen constant; $a_{i}^{\mathrm{L}}, a_{i}^{\prime}$ are the activities of the $i$ th element in the liquid and the interface layer, respectively, which can be calculated by eqs. (7) and (8):

$$
\begin{gathered}
R T \ln a_{i}^{\mathrm{L}}=R T \ln x_{i}^{\mathrm{L}}+G_{\mathrm{m}}^{\mathrm{E}}+\sum_{j=2}^{r}\left(\delta_{i j}-x_{j}\right) \frac{\partial G_{\mathrm{m}}^{\mathrm{E}}}{\partial x_{j}}, \\
G_{\mathrm{m}}^{\mathrm{E}}=(1-f) G_{1}^{\mathrm{E}}+f G_{\mathrm{c}}^{\mathrm{E}},
\end{gathered}
$$

where $G_{\mathrm{m}}^{\mathrm{E}}$ is the excess Gibbs energy of the system; $G_{\mathrm{c}}^{\mathrm{E}}, G_{1}^{\mathrm{E}}$ are the excess Gibbs energies of the clusters and of the residual melt, respectively, whose compositions can be obtained by the conservation of mass; $f$ is the molar fraction of the clusters in the melt; subscripts $i, j$ represent the $i$ th and $j$ th element, respectively, when $i \neq j, \quad \delta_{i j}=0$ and when $i=j, \delta_{i j}=1$.

\section{Results and discussion}

\subsection{Effect of clusters on Gibbs free energy}

Based on eqs. (3), (5) and (6), the Gibbs free energies of $\mathrm{Zr}-\mathrm{Al}-\mathrm{Ni}$ alloy melt with $\mathrm{Zr}_{2} \mathrm{Ni}$ clusters and with free atoms at $T=1000 \mathrm{~K}$ were calculated, which are shown in Figure 1. Comparison of Figure 1(a) and (b) shows that the Gibbs free energy of the melt with the clusters decreases obviously in the composition range of $x_{\mathrm{Ni}}=0.2-0.6$ and $x_{\mathrm{Al}}=0-0.4$, which will cause the decrease of the driving force for nucleation during the solidification due to the decrease of the free energy difference between the liquid phase and solid phase. As a result, the melt with the $\mathrm{Zr}_{2} \mathrm{Ni}$ clusters is favorable to the formation of metallic glass. Compared with the existing experimental results ${ }^{[15]}$, the above composition range is found exactly in the range where metallic glass can be obtained easily.

The Gibbs energies of $\mathrm{Zr}_{66} \mathrm{Al}_{8} \mathrm{Ni}_{26}$ alloy melt with the $\mathrm{Zr}_{2} \mathrm{Ni}$ clusters and with free atoms are shown in Figure 2. Obviously, the melt with the clusters has lower Gibbs energy than that with free 


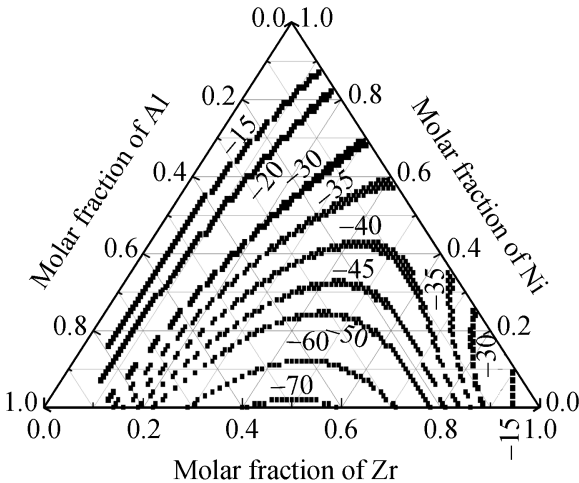

(a)

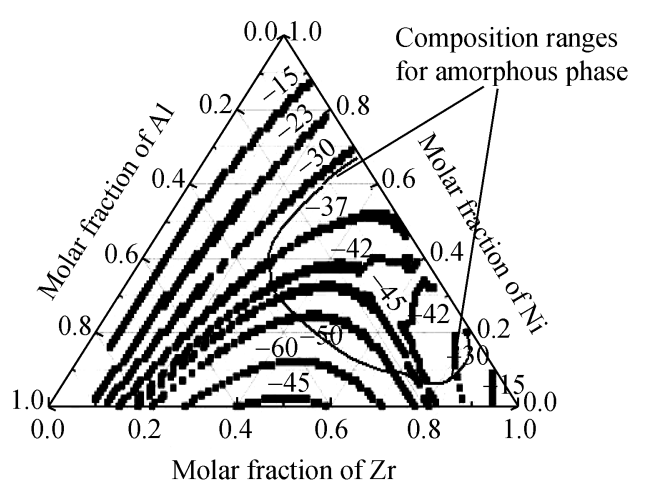

(b)

Figure 1 Gibbs energy distribution of $\mathrm{Zr}-\mathrm{Al}-\mathrm{Ni}$ alloy melt $(T=1000 \mathrm{~K})$. (a) Melt with free atoms; (b) melt with $\mathrm{Zr}_{2} \mathrm{Ni}$ clusters.

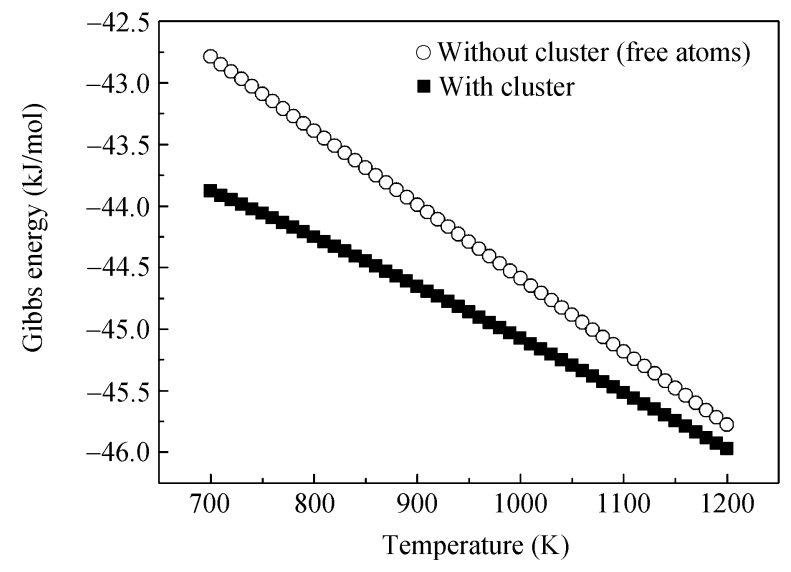

Figure 2 Gibbs energy of $\mathrm{Zr}_{66} \mathrm{Al}_{8} \mathrm{Ni}_{26}$ alloy melt.

atoms, which is more distinct at lower temperature. The Gibbs energy of $\mathrm{Zr}_{66} \mathrm{Al}_{8} \mathrm{Ni}_{26}$ alloy will drop about $0.3-1 \mathrm{~kJ} / \mathrm{mol}$ according to the results of the existence of the clusters.

The calculations show that the quantity of clusters forming in alloy melt is dependent on temperature, and their relationship is shown in Figure 3. It is clear that the molar fraction of the clusters in the melt increases with the decrease of temperature. This is due to the formation of the clusters which is related not only to the free energy difference between the melt and clusters but also to the mobility of atoms. At higher temperature, the free energy difference is small, which means the nucleation driving force is small, and the probability of forming clusters by atom collision is also small due to strong atom mobility. With temperature decreasing, the number of the clusters gradually increases because of the increase of the nucleation driving force and the decrease of the atom mobility. Therefore, at higher temperature, there is smaller effect of clusters on the free energy, while the effect of clusters becomes very evident at lower temperature.

\subsection{Effect of clusters on interfacial energy}

The melt/nuclear interfacial energies were calculated for the $\mathrm{Zr}$-Al-Ni ternary alloy melt with $\mathrm{Zr}_{2} \mathrm{Ni}$ clusters and with free atoms, respectively, whose distributions at $1000 \mathrm{~K}$ are shown in Figure 4. 


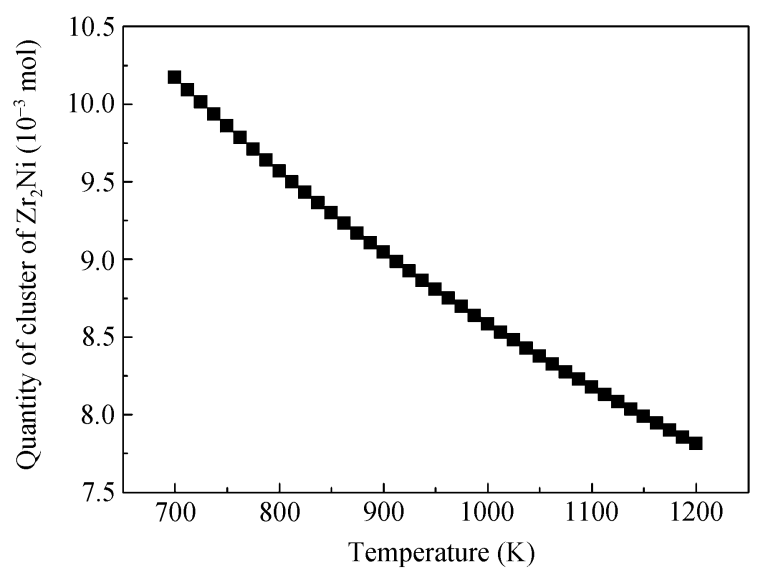

Figure 3 Quantities of $\mathrm{Zr}_{2} \mathrm{Ni}$ clusters in $\mathrm{Zr}_{66} \mathrm{Al}_{8} \mathrm{Ni}_{26}$ alloy melt.

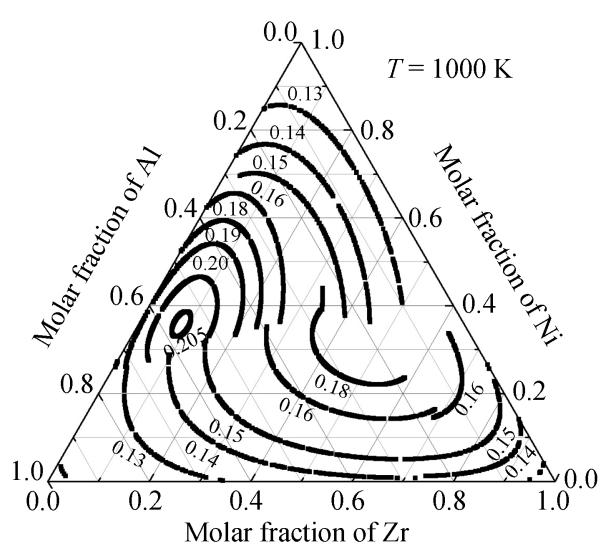

(a)

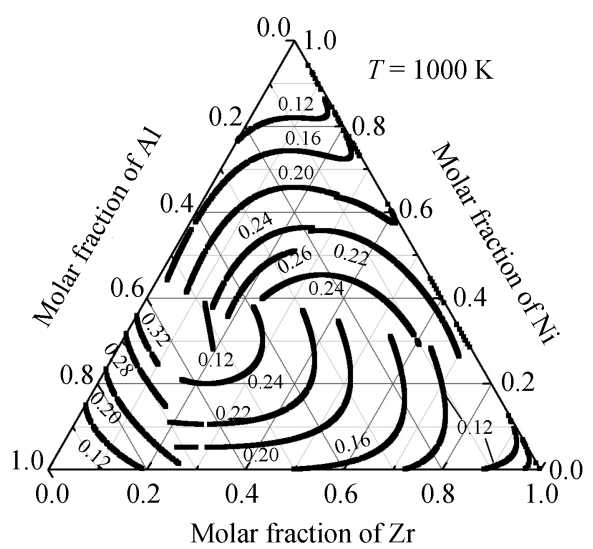

(b)

Figure 4 Interfacial energy in the $\mathrm{Zr}-\mathrm{Al}-\mathrm{Ni}$ alloy melt ( $T=1000 \mathrm{~K}$ ). (a) With free atoms; (b) with $\mathrm{Zr}_{2} \mathrm{Ni}$ clusters.

The contour lines of the interfacial energy in the figure are discontinuous, and the interfacial energy is higher near the eutectic boundaries due to the different precipitated phases in different concentration ranges. The increase of interfacial energy is caused by the concentration of components and configuration entropy increasing near the eutectic boundaries. In addition, the closer the composition of alloy to the eutectic, the higher the interfacial energy, which is consistent with the conclusion that the amorphous can be easily obtained near the eutectic composition.

Through comparing Figure 4(a) with Figure 4(b), we see that not only the interfacial energy distribution with the clusters is changed, but also the interfacial energy of the melt with $\mathrm{Zr}_{2} \mathrm{Ni}$ clusters is higher than that with free atoms (Figure 4(a)) at the same temperature and with the same composition.

The calculation results of the interfacial energy of the $\mathrm{Zr}_{66} \mathrm{Al}_{8} \mathrm{Ni}_{26}$ alloy melt with $\mathrm{Zr}_{2} \mathrm{Ni}$ clusters and with free atoms at different temperature are shown in Figure 5. It can be seen that whether the melt with or without the $\mathrm{Zr}_{2} \mathrm{Ni}$ clusters, the interfacial energy increases with the increase of temperature. The existence of the clusters makes the interfacial energy increase about $0.016 \mathrm{~J} / \mathrm{m}^{2}$ at $1000 \mathrm{~K}$. 


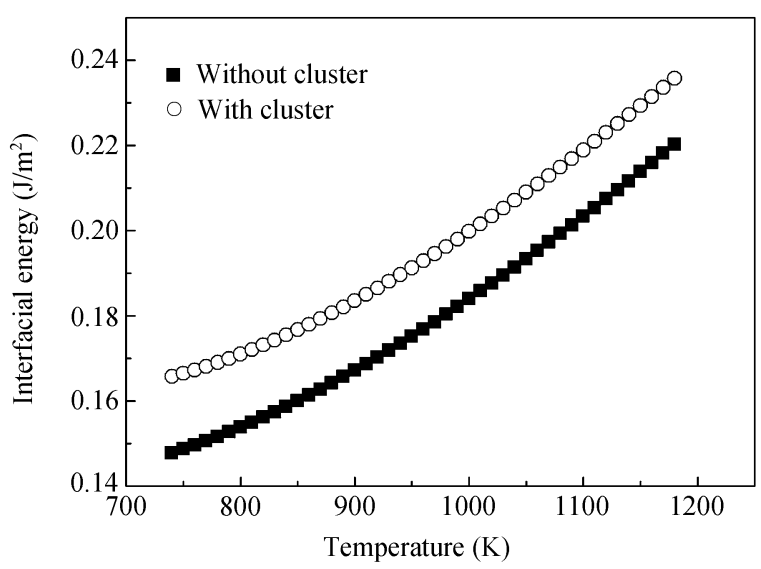

Figure 5 Interfacial energy of $\mathrm{Zr}_{66} \mathrm{Al}_{8} \mathrm{Ni}_{26}$ alloy melt.

\subsection{Effect of clusters on nucleation of melt}

The nucleation rates of the undercooled $\mathrm{Zr}_{66} \mathrm{Al}_{8} \mathrm{Ni}_{26}$ melt with clusters and undercooling, $\Delta T$, are calculated as shown in Figure 6, in which curve (1) represents the nucleation rate with free atoms, and curves (2), (3), (4) are the corresponding nucleation rate, respectively, with the free energy, the interfacial energy and the total energy influenced by formation of clusters.

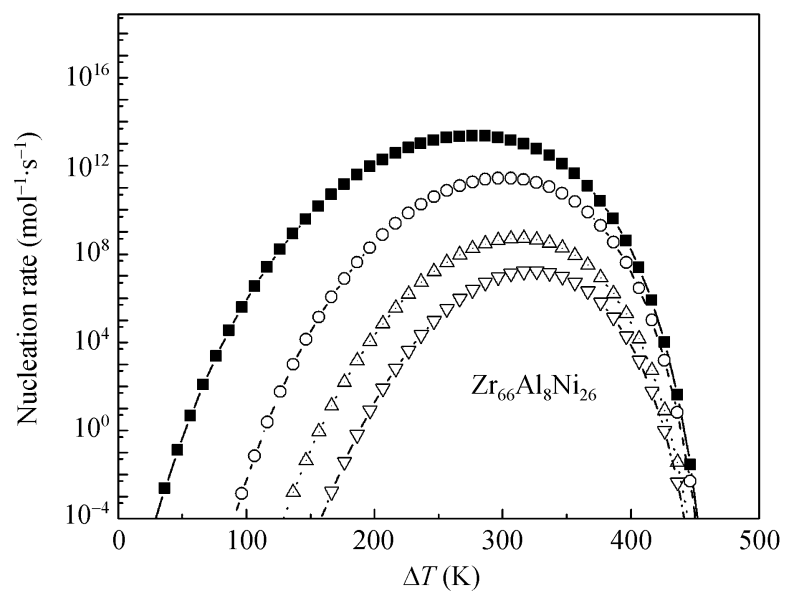

Figure 6 The nucleation rates of $\mathrm{Zr}_{66} \mathrm{Al}_{8} \mathrm{Ni}_{26}$ melt. $\mathbf{\square}$, Nucleation rate (1); $\circ$, effect of Gibbs energy with cluster (2); $\triangle$, effect of interface energy with cluster (3); $\nabla$, effect of Gibbs energy and interface energy with cluster (4).

According to curve (1), the nucleation in the $\mathrm{Zr}_{66} \mathrm{Al}_{8} \mathrm{Ni}_{26}$ alloy melt with free atoms happens when $\Delta T=40 \mathrm{~K}$ and the maximum nucleation rate reaches $2.26 \times 10^{13} \mathrm{~mol}^{-1} \cdot \mathrm{s}^{-1}$ with $\Delta T=270 \mathrm{~K}$, then it decreases with $\Delta T$ increasing. This is because the viscosity of the melt is much higher than that of common alloys, and the nucleation needs larger driving force of larger undercooling. The diffusion of the atoms in the melt is more difficult at lower temperature due to the melt viscosity dramatically increasing with the increase of $\Delta T$, so that the nucleation rate decreases when $\Delta T$ is too large.

The effect of the melt Gibbs energy with the clusters on the nucleation rate can be seen by comparing curve (2) with curve (1). When the clusters exist in the alloy melt, the nucleation rate 
decreases obviously or the undercooling for nucleation increases. This is because that the Gibbs energy of the melt is dropped by the $\mathrm{Zr}_{2} \mathrm{Ni}$ clusters, which causes the decrease of the nucleation driving force. In contrast, the effect of the interfacial energy with the existence of the clusters on the nucleation rate is more evident, as shown in curve (3), and at this condition, much larger undercooling is needed for the initial nucleation because the formation of the interface is more difficult due to the increase of the interfacial energy. Curve (4) combines both effects of the change of Gibbs energy and interfacial energy, i.e., the superimpose of curves (2) and (3). It can be seen that the nucleation rate in the melt with the clusters evidently decreases, and the maximum nucleation rate is only in the order of $10^{7}$. In addition, the undercooling for initial nucleation increases to $160 \mathrm{~K}$ and the undercooling range for nucleation decreases from $410 \mathrm{~K}$ to $290 \mathrm{~K}$.

\section{Conclusions}

(1) $\mathrm{The}_{\mathrm{Zr}} \mathrm{Ni}$ clusters in the melt of $\mathrm{Zr}-\mathrm{Al}-\mathrm{Ni}$ alloy make the Gibbs energy decrease in the composition range where metallic glass can easily form. For the $\mathrm{Zr}_{66} \mathrm{Al}_{8} \mathrm{Ni}_{26}$ alloy melt, the Gibbs energy decreases about $0.3-1 \mathrm{~kJ} / \mathrm{mol}$.

(2) $\mathrm{The} \mathrm{Zr}_{2} \mathrm{Ni}$ clusters can result in the increase of the interfacial energy of the $\mathrm{Zr}-\mathrm{Al}-\mathrm{Ni}$ alloy melt, and change the distribution of the interfacial energy with the composition. The interfacial energy of the $\mathrm{Zr}_{66} \mathrm{Al}_{8} \mathrm{Ni}_{26}$ alloy melt with the clusters is about $0.016 \mathrm{~J} / \mathrm{m}^{2}$ higher than that of the melt with free atoms.

(3) $\mathrm{The} \mathrm{Zr}_{66} \mathrm{Al}_{8} \mathrm{Ni}_{26}$ alloy melt with $\mathrm{Zr}_{2} \mathrm{Ni}$ clusters has lower nucleation rate and needs larger undercooling for initial nucleation than the melt with free atoms. The maximum nucleation rate of the $\mathrm{Zr}_{66} \mathrm{Al}_{8} \mathrm{Ni}_{26}$ alloy melt is $10^{7} \mathrm{~mol}^{-1} \cdot \mathrm{s}^{-1}$, the initial nucleation undercooling is $160 \mathrm{~K}$ and the undercooling range for nucleation is $290 \mathrm{~K}$.

1 Turnbull D. Under what conditions can a glass be formed? Contemp Phys, 1969, 10(5): 473-488

2 Uhlmann D R. A kinetic treatment of glass formation. J Non-Cryst Solids, 1972, 7: 337-348

3 Wei Q C. Thermodynamics in Metallurgy (in Chinese). Chongqing: Chongqing University Press, 1996. $73-91$

4 Goken N A. Statistical thermodynamics of alloys. New York: Plenum Press, 1986. 255-273

5 Li H Q, Yang Y S, Tong W H, et al. Calculation of Gibbs energy of Zr-Al-Ni, Zr-Al-Cu, Al-Ni-Cu and Zr-Al-Ni-Cu liquid alloys based on quasiregular solution model. J Alloys Compds, 2007, 428(1-2): 185-189

6 Saida J, Matsushita M, Inoue A. Stability of supercooled liquid and transition behavior in Zr-based glassy alloys. Mater Trans, 2002, 43(8): 1937-1946

7 Frank F C. Supercooling of liquids. Proc Royal Soc London A, 1952, 215(1120): $43-46$

8 Hao J A, Zheng H P. Theoretical calculation of structures and properties of $\mathrm{Ga}_{6} \mathrm{~N}_{6}$ cluster. Acta Phys Sin-CH ED (in Chinese), 2004, 53(4): 1044-1049

9 Li G P, Zhang M L. Energetics and structures of high-temperature copper cluster studied by Monte Carlo method. Acta Phys Sin-CH ED (in Chinese), 2005, 54(6): 2873-2876

10 Wang Y, Li P, Ning X J. Molecular dynamics study on self-assembly of C36 clusters. Acta Phys Sin-CH ED (in Chinese), 2005, 54(6): 2847-2852

11 Desré P. Thermodynamic analysis of concentration fluctuations and homogeneous nucleation of crystal in undercooled liquid binary alloys: Application to glass forming ability. J Mater Sci, 1987, 22: 57-62

12 Chen G L, Hui X D, Fan S W, et al. Concept of chemical short-range-order domain and the glass forming ability in multicomponent liquid. Intermetallics, 2002, 10: 1221-1232

13 Holland-Moritz D. On the energy of the interface between a melt and quasicrystalline and polytetrahedral phases. J Non-Cryst Solids, 1999, 250-252: 839-843

14 Li H Q, Yang Y S, Tong W H, et al. Calculation of solid-liquid interfacial energy for Zr-Ni-Al and Zr-Ni-Al-Cu alloys based on non-structural approach. Modeling Simul Mater Sci Eng, 2006, 14: 1095-1103

15 Inoue A, Zhang T, Masumoto T. Glass-forming ability. J Non-Cryst Solids, 1993, 156-158: 473 - 480 